\title{
Decomposition of Image Restoration Model in the Application of Tibetan Ancient Thangka Repair
}

\author{
Jun Jiang ${ }^{12}$ \\ Colledge of Engineering ,Tibet University, Lhasa 850000, China \\ E-mail: $32874920 @$ qq.com

\section{Zhaoxia Wang ${ }^{3}$} \\ Department of Student ,Tibet University, Lhasa 850000, China \\ E-mail: $124442105 @$ qq. com
}

\begin{abstract}
This paper analyzes the classical integral variational denoising algorithm and the image repaire algorithm, which is put forward based on the structure-decomposition of the texture image retrieval algorithms. Algorithm will firstly part to repair the image which is decomposed into structure and texture details, and the imperfect parts of the image contour structure of the contour structure repair and vacancy of texture details of the image texture synthesis. The final will be repaired after the part structure and texture details to carry on the synthesis so as to get the ideal image finally. The experimental results show that the algorithm has very good repair effect.

On basis of analyzing the classical integral variational denoising algorithm and image repairing algorithm, this paper puts forward an image retrieval algorithm based on the decomposition of the structure-the texture. In this algorithm, the image to be repaired is firstly decomposed into the outline structure part and the texture detail part, and then the imperfect part of the outline structure is repaired and the vacancy of the details of the texture image is synthesized. Finally, an ideal repaired image can be obtained by synthesis of the repaired structure part and the texture detail part. The experimental results show that the algorithm has a very good repair effect.
\end{abstract}

\footnotetext{
${ }^{1}$ Speaker

${ }^{2}$ Corresponding Author

${ }^{3}$ The research was sponsored by the National Natural Science Fund (Project No.: 61461047), the National Natural Science Foundation of Tibet Autonomous Region in 2012 (Project No.: Z2012Z01G07), and the 2015 youth backbone teacher of Everest Scholars Talent Development Support Program of Tibet University.
} 


\section{Introduction}

Tibetan Thangka features a very long history. As the national heritage is usually influenced by both natural and anthropogenic factors, there are plenty of Thangka damaged, polluted and bleached. Thangka repair is urgent. Artificial restoration which is irreversible, risky and high costs has become a major obstacle to Thangka repair. Digital mural restoration technology can not only save the mural picture permanently but minimize the risk and investment through a PC simulation of artificial restoration process which can repair the damaged pictures fictitiously.

With the experience of restoring the ancient artist's art works in [1] [2], modern image restoration technology is mainly based on such two aspects of the structure and the texture repair. The part of structure mainly describes the main changes of the subject and the part of texture mainly describes the local details. The image's gradient, level set, level and curvature are the main considerations of image structure repair. Chan proposed the non-textured curvature drive repair model(CDD:Curvature Driven Diffusions) in [3], which has a typical representative of significance in larger deletions structure repair. The image's texture, pixel and region similarity are the key factors of image texture repair. The texture synthesis is mainly based on the texture synthesis algorithm as proposed by Criminisi in [4].

On the basis of studying these two algorithms, the paper introduced them to the Tibetan Thangka repair, which can effectively recover the missing part of the outline of the image and the corresponding texture details.

\section{Image Decomposition}

As both the image structure repair and the texture synthesis process rely on the image decomposition, which is the most critical one in the whole process. Decomposition needs using two kinds of image decomposition techniques. One is based on the overall scale variational model in [5]. According to the image scale to be obtained, the appropriate regularization parameter should be selected. The selection basis is that the large-scale image of the regularization parameters should correspond to large-scale image and small-scale image of small regularization parameters correspond to small. The other is the image decomposition algorithm proposed by Vese in [6]. This decomposition method introduces an oscillating function space proposed by Y. Meyer to represent the texture, which can separate the texture and contour structure better. In order to get better results in the image repair, this algorithm is adopted to decompose the image. The image decomposition algorithm proposed by Vese was developed by Total Variation Denoising. It introduced the oscillation function space to model texture and noise, which is different from Total Variation Denoising Algorithm.

Assume $I: R 2 \rightarrow R$ denotes the observed real images and $I \in L 2(R 2)$ is the decomposition of the object image which can be noisy. $u$ represents the structure or profile image obtained upon decomposition of $I$ and $v$ represents the texture detail images upon decomposition of I (possibly noisy). There is a linear relation between $\mathrm{I}, \mathrm{u}$ and $\mathrm{v}$, as follows:

$$
I(x, y)=u(x, y)+v(x, y)
$$

The total variation model can be transformed

$$
\operatorname{in}_{u \in B V} f\left\{E(u)=\int \nabla u \mid \lambda\|\|_{V} \|_{L^{2}}^{2} ; I=u+V\right\}
$$

In the Eq. (2.2), BV represents bounded variation function space. Item 1 in the Eq. (2.2) is the regularization term, which works in noise remove and texture detail. 


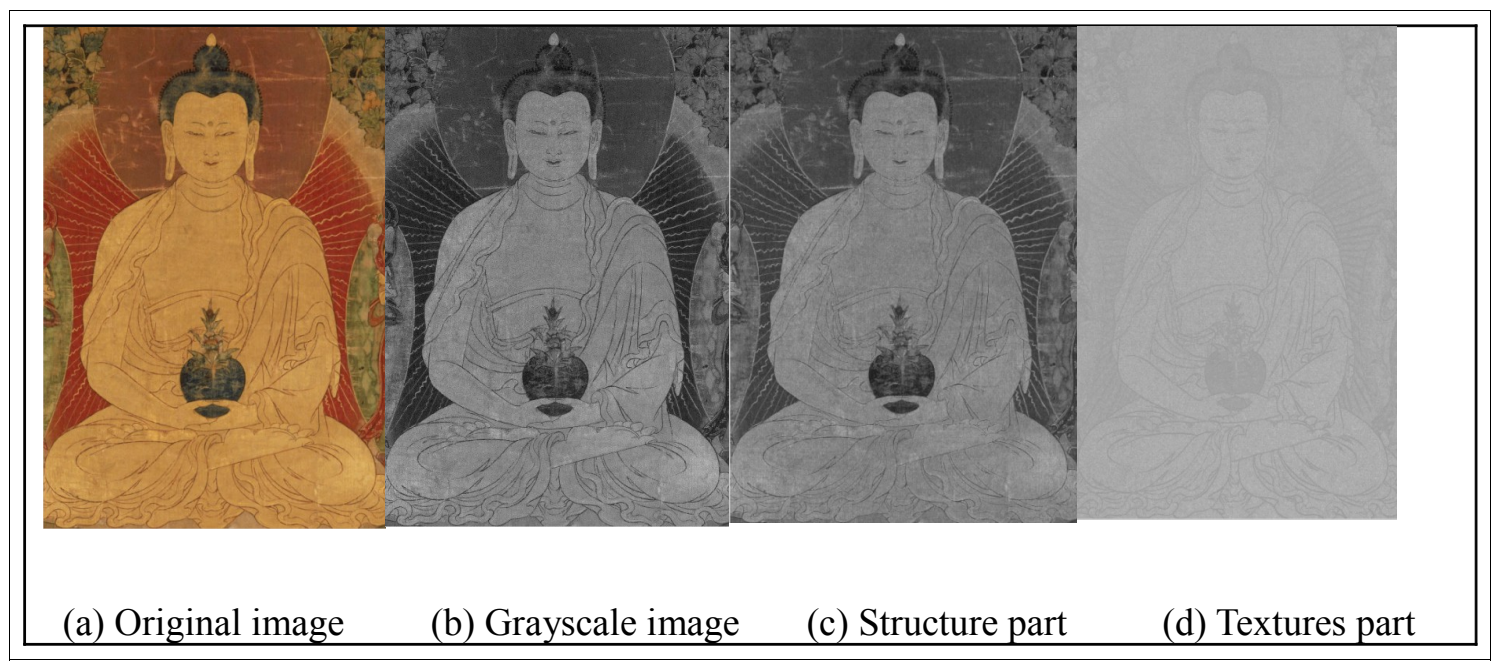

Figure 1: Image Decomposition

\section{Subgraph Repair}

\subsection{Structure of the Image Repair}

Compared with other models of partial differential equations, TV models adopt a secondorder partial differential equations. It is relatively simple to implement and converges fast, but it always connects broken stripe structure with the shortest line, which is likely to cause distortion. In order to solve this problem, Tony. Chan and Shen et al proposed the idea that curvature promotes proliferation, also called CDD model in [7], which introduces the curvature term further based on the TV repair model by adding a penalty term $\mathrm{g}(\mathrm{k})$ to the diffusion equation. That means diffusion is larger when curvature is larger in [8], in this sense, the overall balance can be obtained. Whereby:

$$
E_{C D D}\left[u \mid u_{0}, D\right]=\iint_{\Omega} g(k)|\nabla u| d x d y+\frac{\lambda t}{2} \iint_{\frac{D}{\Omega}}\left|u-u_{0}\right| d x d y
$$

Where, $\mathrm{k}$ is the horizontal curvature and $\mathrm{g}(\mathrm{k})$ is a monotonically increasing function of the curvature. And

$$
\mathrm{g}(k)=\left\{\begin{array}{l}
0 \quad, k=0 \\
\infty \quad, k=\infty \\
\text { other } \quad \text { value }, 0<k<\infty
\end{array}\right.
$$

By solving it with the discrete method, we can obtain the following iterative formula:

$$
\begin{aligned}
& j=\left(j^{1}, j^{2}\right)=\frac{g(|k|) \nabla u}{|\nabla u|} \\
& u^{n+1}=u^{n}+\Delta t \bullet \nabla j^{n}
\end{aligned}
$$

where, $\Delta \mathrm{t}$ is the time step and $\mathrm{u}^{\mathrm{n}}$ represents a value when the repair point is $\mathrm{n} \Delta \mathrm{t}$.

CDD repair model's diffusion strength depends on not only the gradient, but also the Isolux line's geometry information, namely the curvature in [9]. Since $g$ is an increasing function, diffusion is stronger when Isolux line's curvature is larger and gradually weaken when the Isolux line extenses. This makes it possible to eliminate the large curvature and stable small curvature in the repair process and it's in the line with the "overall connectivity criterion". 


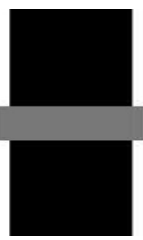

(a) To be repaired

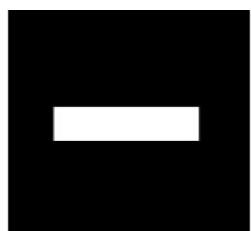

(b) MASK

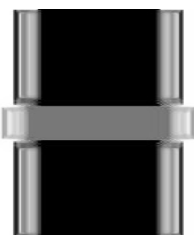

(c) TV repair

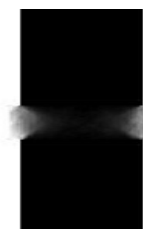

(d) CDD repair

Figure 2: Comparison of the Overall Connectivity

As can be seen from Figure 2, CDD model meets the basic overall repair connectivity; therefore, the CDD law's repair ability is stronger than that of the TV law, which can repair the larger damaged area and the smaller fracture edges.

\subsection{Texture Repair}

With the better synthetic, the block-based texture synthesis algorithm proposed by Criminisi has been extensively studied. Later, the vast majority of researchers have adopted the basic framework of image patch proposed from this algorithm in [10]. The basic process of repair methods is mainly made up with three steps, namely, priority calculation, searching and copying. Before repair, the image partition is shown in Figure 3(a), which represents the loss of information area to be patched in the image. $\Omega$ represents the known portion whose information hasn't been lost in the image and $\delta \Omega$ is the border surrounding the area to be patched. The first step of priority calculation is mainly to determine the repair order, which is the target block to be repaired at first. As shown in Figure 3(b), it calculates the priorities of points' to be repaired on the boundary region firstly and selects the point $\mathrm{p}$ which has the highest priority. And then it determines the target block $\Phi p$ need to patch with point $\mathrm{p}$ centered. For the purpose identification, area $\Omega$ is recorded as $\Phi p$ and area $\Phi$ is recorded as $\Phi p$, both belonging to $\Phi p$.

Step 2 is to search. It should search the matching block $\Phi$ p of in $\Phi$ 's image known region according to the texture feature of in $\Phi p$ target block $\Phi$ p. As shown in Figure 3(c), the resulting optimal matching block is $\Phi \mathrm{q}$.

Step 3 is to copy. It should copy the best matching bloc $\Phi p \mathrm{k}$ and the corresponding part of $\Phi p$ into , $\Phi p$ which makes $\Phi p$ to get patched and updates boundaries of area to be patched $\delta \Omega$, as shown in Figure $3(\mathrm{~d})$.

In order to complete the repair of an image block through the three-step operation, repeating these three steps can fill the area to be repaired and complete the picture's patch.

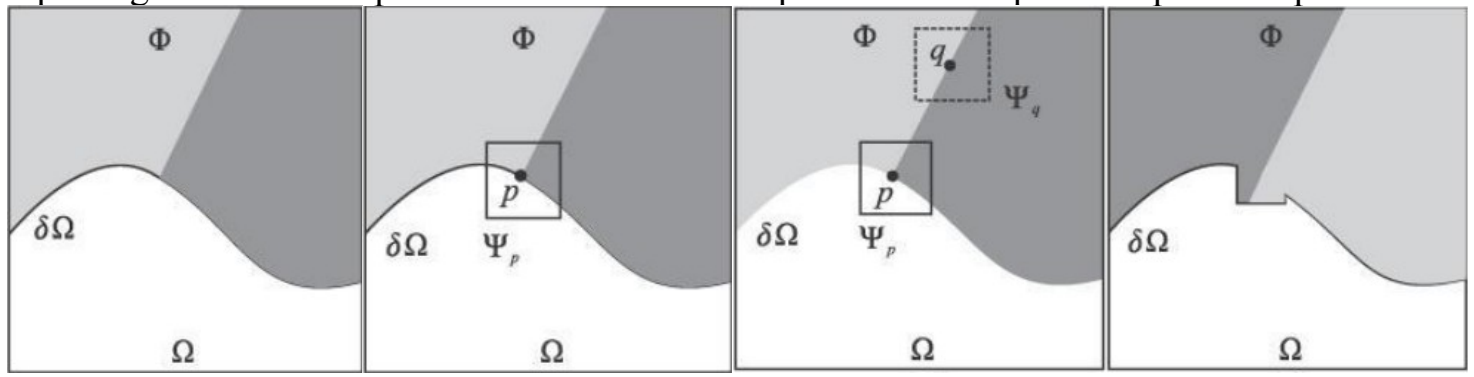

(a)

(b)

(c)

(d)

Figure 3: Model for the Repair 


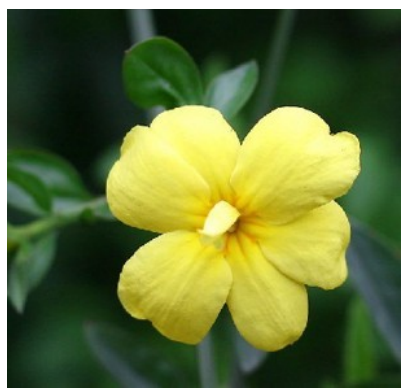

(a)The original image

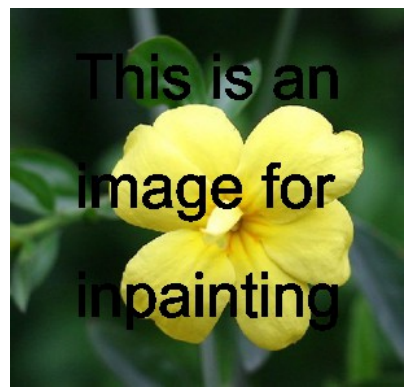

(b) Damaged image

PSNR=33.25 Repair elapsed time 455.92 seconds

Figure 4: Repair Damaged Small Area

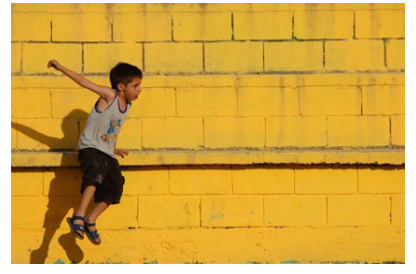

(a)

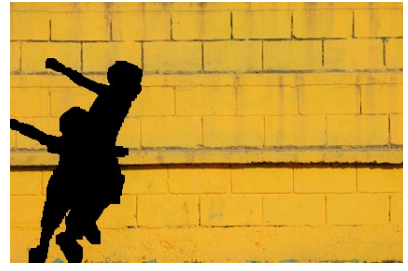

(b)

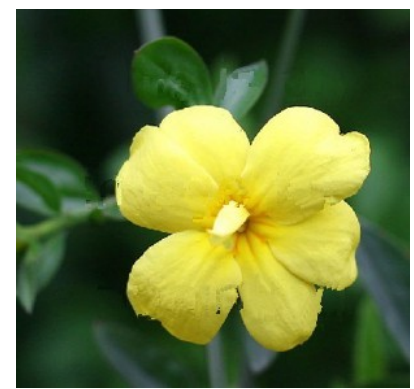

(c)The restored image

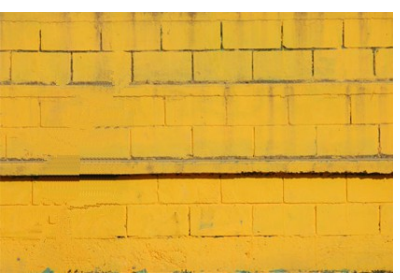

(c) Repair elapsed time 495.84 seconds

Figure 5: Repair Damaged Big Area

\section{Results and Discussion}

Firstly, it should mark the original image's portion to be repaired with a white bold line, and then divide the original image after decomposing it into the outline structure image and texture detail image.

The RGB(Red,Green ,Blue) image is decomposed into three channels of $R, G, B$ in structure part by using the decomposition idea. Then each channel is restored one by one. Finally, these three channels are combined and the repair is completed.

According to the information around the area to be repaired, it should choose a texture image which has the main grain direction as an input image in textures part. An edge image of the inputted image is obtained with sobel edge detector. The direction of the edge image, which is the main direction of the texture is obtained through the Fourier transform. The boundary points of the target area are extracted. Each point corresponding to the pixel block by the default window size is determined and their priority is calculated. The first pixel to be filled is determined. A new template's size is obtained according to the Gradient. The new template is adopted to search the most similar block along the main grain direction in the source region; then update the priority upon filling the new pixel. Repeat these steps until when the pixels turn to zero in the target area, and thus the image repair is completed.

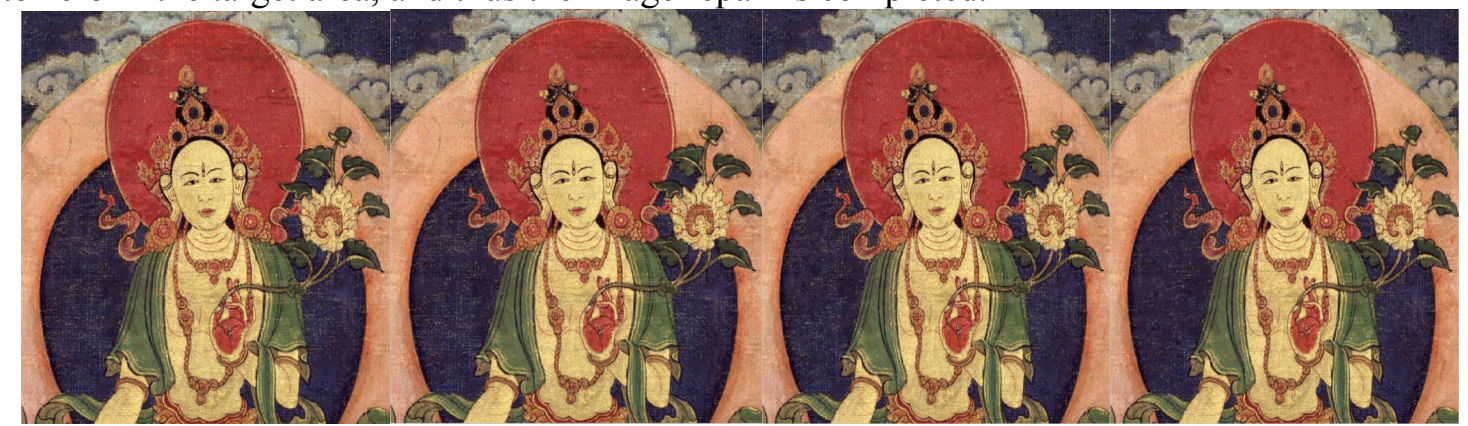

(a)damaged image

(b) CDD algorithm

(c)Criminisi algorithm (d) The paper algorithm

Figure 6: Rpair Results 
Used time (the paper algorithm) is 167.43 seconds. We can get the following results by detecting function: (Abscissa: iterations ordinate: PSNR factor)

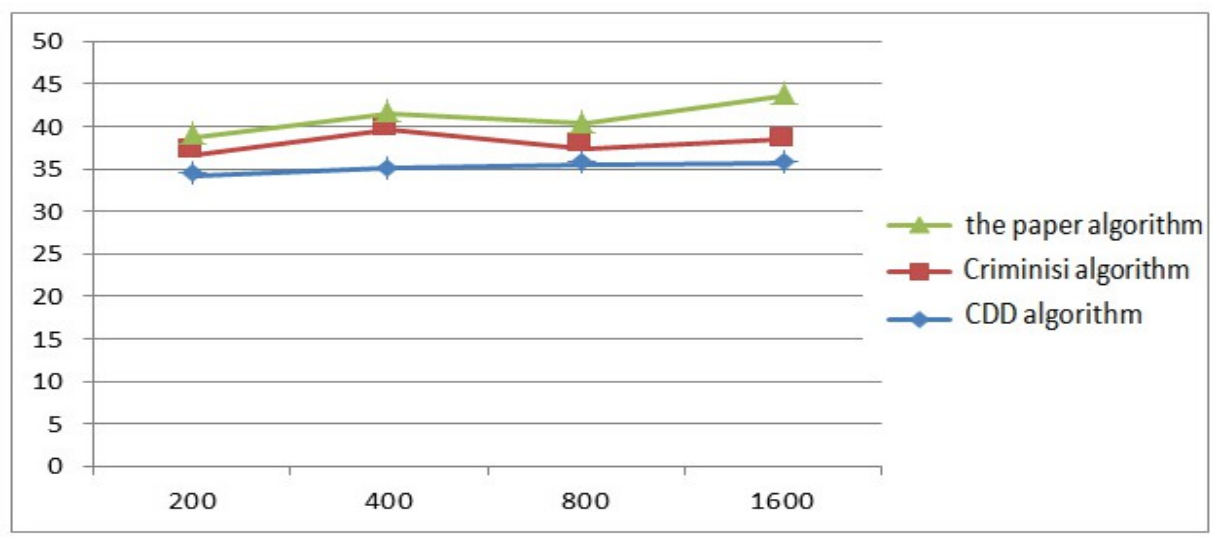

Figure 7: Contrast Table of the Evaluation Function

From Figure 7, we can see that as to the repair effect, this model is obviously superior to the independent repair model. The effect of image restoration is getting better with the increase of the number of iterations.

\section{Conclusion}

Experimental results show that decomposition algorithm is better than the pure texture synthesis and the pure structural repair technique, primarily in that it considers the texture detail and the structure contour of the damaged image together in the process of patching algorithm; besides, it also uses the neighboring texture and structure information of the area to be repaired according to a certain pattern so as to fill the area to be repaired. The pure texture synthesis techniques only considers the texture information while not considering the relationship between the image structure of the region to be repaired and the neighborhood's in a comprehensively manner. Similarly, although the pure structure repair technique can repair structure information of the area to be repaired, it is not related to more detailed texture information; therefore, the basic structure can be recovered, but great contrast exits between the overall feeling and the texture reaction in the neighborhood. It's special clear in the image which has rich texture.

Since the algorithm is based on the image texture contour decomposition, a more efficient method to decompose the image to be repaired is the direction of our future research. In addition, although the algorithm allows the presence of noise and has some ability of de-noise, the texture detail image still contains some noise upon decomposition. If the noise is not well separated from the texture, the final synthesized image will still contain some noise.

\section{References}

[1] Hongying Zhang, Qicong Peng. A Survey on Digital Image Inpainting[J].Journal of Image and Graphics. 12( 1), 1-10, 2007 (In Chinese).

[2] Hongying Zhang, Qicong Peng, Yadong WU. Digital Image Inpainting Algorithm for Damaged Images Based on Nonlinear Anisotropic Diffusion [J]. Journal of Computer Aided Design\&Computer Graphics. 18( 1), 1542-1546, 2006 (In Chinese).

[3] Chan T F, Shen J H. Mathematical models for local non-texture inpainting[J].SIAM Journal of App lied Mathematics, 62(7),1019-1043, 2001.

[4] Jun JIANG, Ga ZHUO, Zhao-xia WANG.The Research of Tibet Mural Digital Images inpainting Using TV model[J]. Electronic Design Engineering. 21(3), 136-139, 2013 (In Chinese). 
[5] Shanshan ZHOU, Zhijing ZHAO, Xueyi YE. Total variation and texture synthesis applied to multi-level image inpainting[J]. Computer Engineering and Applications, 47(23), 201-203, 2011 (In Chinese).

[6] Xuan ZHU, Ming quan ZHOU, Guo hua GENG.A New Noise Removal Method Based on Thirdorder Nonlinear Diffusion and its Algorithm Realization[J]. Computer Science. 35(12),212_213, 2007 (In Chinese).

[7] Yanwei ZHAO, Xianglin LI. A Rapid Image Inpainting Algorithm Based on CDD Model[J]. Computer Simulation. 25(10),223-227,2008(In Chinese).

[8] Xiangxin SHAO, Shuxu GUO, Lang WANG. Image Mosaic Algorithm Based on Extended Phase Correlation of Edge[J]. Journal of Jilin University(Information Science Edition). 28(1),95-99, 2010(In Chinese).

[9] Wei HUANG,Shuwen WANG,Xiaoping YANG,Jianfang JIA. Dun huang murals inpainting based on image decomposition[J]. JOURNAL OF SHANDONG UNIVERSITY (ENGINEERING SCIENCE).40(2),24-33, 2010(In Chinese).

[10] Chunyan ZHAO, Yan ZHAO, Hexin CHEN. Registration of Depth and Video Data Based on Edge Detection[J]. Journal of Jilin University(Information Science Edition).29(3),175-180, 2011(In Chinese). 